\title{
Numerical simulation of microstructure evolution on near eutectic spheroidal graphite cast iron
}

\author{
Bing Wu, Di Meng, *Hong-liang Zheng and Xue-lei Tian \\ Key Laboratory for Liquid-Solid Structural Evolution and Processing of Materials, Ministry of Education, Shandong University, Jinan 250061, China
}

\begin{abstract}
A multiphase cellular automaton model was developed to simulate microstructure evolution of near eutectic spheroidal graphite cast iron (SGI) during its solidification process, and both dendritic austenite and spheroidal graphite growth models were adopted. To deduce the mesh anisotropy of cellular automaton method, the composition averaging and geometrical parameter were introduced to simulate the spheroidal graphite growth. Solute balance method and decentered square algorithms were employed to simulate austenite dendrites growth with different crystallographic orientations. The simulated results indicate that the graphite nodule grows in a spherical morphology when the surrounding environment of a single graphite nodule is same. However, for two adjacent graphite nodules, the environment is different. The higher the carbon concentration, the faster the growth of graphite. By comparison with experimental results, it is found that the microstructure evolution of near eutectic spheroidal graphite cast iron during solidification process can be reproduced quantitatively by numerical simulation with this model.
\end{abstract}

Key words: spheroidal graphite cast iron; cellular automaton model; numerical simulation; mesh anisotropy; graphite growth

CLC numbers: TP391.99 Document code: A Article ID: 1672-6421(2017)05-386-06

Qpheroidal graphite cast iron (SGI) has the most extensive application in practice, especially in the automotive industry with its high strength, excellent mechanical properties and competitive price ${ }^{[1,2,3]}$. However, due to the unique solidification characteristics, the defects such as shrinkage cavity and porosity which can affect the mechanical properties of alloys are difficult to avoid in the ductile iron casting. We often reduce the generation of defects by improving the casting method in the macroscopic field. On the other hand, in the microscopic domain, understanding the microstructure morphologies of spheroidal graphite cast iron and controlling their evolution process can better reveal the mechanism and achieve the desired microstructures, and thus control the properties of castings. Similar to the other eutectic alloys, when the temperature is lower than the liquidus temperature, the solidification of SGI starts with the nucleation and growth of primary

\footnotetext{
*Hong-liang Zheng

Male, bore in 1981, Ph.D, Associate Professor. Research interests mainly focus on solidification simulation of nucleation and growth of alloys with micromacro models. More than 20 papers have been published.

E-mail: honglz@126.com

Received: 2017-08-14; Accepted: 2017-09-28
}

phase. Eutectic reaction is triggered once the system temperature drops to the eutectic temperature. For the hypoeutectic SGI, the primary precipitated phase is the austenite which grows in the form of dendrite. Whereas, spherical graphite is the primary solid phase for the hypereutectic SGI.

In order to quantitatively predict the microstructure evolution process of alloy solidification in detail, over the last decades, a number of cellular automaton (CA) technique has been used as a powerful tool. Rappaz and Gandin ${ }^{[4]}$ applied the CA method to solidification simulation, and used the LGK analytical model to simulate the microstructure evolution in Al-Si alloy solidification process. In 1999, Nastac ${ }^{[5]}$ coupled CA model and concentration field firstly. And in this model, a method to solve the concentration at the interface, the calculation of interface curvature and the growth velocity with composition equilibrium approach were proposed. However, the anisotropy of CA method still remained in this study. On the basis of this model, a lot of work has been done to eliminate the artificial anisotropy caused by the CA square mesh. BeltranSanchez and Stefanescu ${ }^{[6]}$ developed a BBS model to simulate the dendrite growth with the axis or diagonal direction of cell. Subsequently, Beltran-Sanchez and 
Stefanescu ${ }^{[7]}$ presented a new rule to capture the interface cell by its normal direction and solid fraction. This method can eliminate anisotropy effectively. But, the shortage of the model is that dendrites have a tendency to grow along the axial direction of grid near the boundary. In 2003, Wang et al. ${ }^{[8]}$ proposed a decentered square algorithm and obtained a good effect on the columnar and equiaxed dendrite simulation in the two-dimensional and three-dimensional domain.

While in the process of using the CA method to simulate the microstructure evolution of SGI, Zhu et al. ${ }^{[9]}$ proposed a multiphase CA model to simulate the divorced eutectic solidification process of SGI. Local solute equilibrium approach was adopted in this model to calculate the driving force for the growth of austenite dendrite and graphite. However, the previous simulation research mainly focused on the study of dendrite, there is little work on the elimination of anisotropy of graphite.

In this work, a new growth model of graphite was established based on the solute equilibrium method. By introducing geometrical parameter and composition averaging which is determined by simulating the growth of a single graphite nodule under different parameters to make the graphite nodule grow in spherical shape. Then, the model couples with dendrite growth model to establish the ductile iron solidification model. Compared with the experimental results, the model can better reproduce the microstructural evolution process during divorced eutectic solidification of SGI.

\section{Model description and numerical algorithm}

\subsection{Description of model}

To better understand the solidification process of SGI, the microstructure of hypoeutectic and hypereutectic SGI were simulated respectively in this study. The computation domain is divided into uniform square grids, in which a grid represents a CA cell. The state of each cell is defined by one of austenite solid phase, graphite solid phase, liquid phase, austenite/liquid interface cell, graphite/liquid interface cell, austenite/graphite/ liquid interface cell and austenite/graphite interface cell. During the period of growth, initially, the state of cells is liquid. When the temperature drops down to the liquidus, a certain number of nuclei of graphite or austenite are randomly distributed in the computation area and the cell state is changed into the interface cell. Then, with the increase of solid fraction of the interface cell, according to the capturing rules, the new liquid cells are captured and transformed into interface cells. Moreover, the state of interface cell changes into solid when the solid fraction is greater than 1 . When the temperature drops below the eutectic temperature, the graphite and austenite grow at the same time, that is, the divorced eutectic solidification process. Among them, the interface capturing rule and the calculation of solid fraction which is related to the temperature field and the concentration field are becoming more important in the simulation.

\subsection{Solute and temperature field algorithm}

The growth of graphite and austenite is mainly controlled by the diffusion of the solute. It is assuming that the natural forced convection is not considered in the solidification process. Considering the solute redistribution in the solidification process of austenite dendrites and graphite, the governing equation for solute field is as follows ${ }^{[10]}$ :

$$
\frac{\partial C_{i}}{\partial t}=D_{i} \nabla^{2} C_{i}+C_{\mathrm{L}}\left(1-k_{\gamma}\right) \frac{\partial f_{\mathrm{S}, \gamma}}{\partial t}+\left(C_{\mathrm{L}}-C_{\mathrm{Gr}}\right) \frac{\partial f_{\mathrm{S}, \mathrm{Gr}}}{\partial t}
$$

where $C_{\mathrm{i}}$ is the composition and the subscript $i$ indicates liquid, austenite $(\gamma)$ and graphite $(\mathrm{Gr}), t$ is the time step, $D_{\mathrm{i}}$ is the diffusion coefficient of the corresponding phase. The second term on the right side of the equation denotes the amount of rejection of carbon during the process of austenite growth and $C_{\mathrm{L}}$ is the composition of interface cell, $k_{\gamma}$ is the equilibrium partition coefficient. $f_{\mathrm{S}, \gamma}$ is the austenitic solid fraction, the subscript $S$ represents the solid phase. Similarly, the third term on the right side of the equation represents the amount of absorption of carbon because of the graphite growth. $C_{\mathrm{Gr}}$ is the solubility of carbon in the graphite phase. $f_{\mathrm{S}, \mathrm{Gr}}$ is the solid fraction of graphite.

In this model, the temperature field consists of two parts. First of all, natural cooling will lead to the decrease of temperature. Then, during the solidification process, due to the phase transformation, a plenty of latent heat is released, which cause the recovery of temperature. While the former is the driving force of solidification. Assuming the temperature in the computation domain is uniform, thus, the temperature field is calculated by ${ }^{[11]}$.

$$
\frac{\partial T}{\partial t}=\dot{T}+\left(\frac{L_{\gamma}}{\rho C_{\mathrm{p}}} \cdot \frac{\partial f_{S, \gamma}}{\partial t}+\frac{L_{\mathrm{Gr}}}{\rho C_{\mathrm{p}}} \cdot \frac{\partial f_{\mathrm{S}, \mathrm{Gr}}}{\partial t}\right) / N^{2}
$$

where $T$ is the real temperature on the domain. $\dot{T}$ is the cooling rate, which refers to the heat exchange between casting and air through the medium of the mold. The second term on the right side of the equation indicates that the released latent heat is evenly distributed to all the cells. $L_{\gamma}$ and $L_{\mathrm{Gr}}$ are the volume latent heat of the austenite and graphite during solidification. $\rho$ is the density and $C_{\mathrm{p}}$ is the specific heat of the liquid. $N^{2}$ denotes the total number of cells in the area.

\subsection{Growth algorithm of austenite and graphite}

In this study, a solute balance method is adopted to calculate the solid fraction at each time step. Its principle is that using the difference between the actual concentration and the equilibrium concentration of the interface cells calculates the solid fraction. The governing equations for austenite and graphite growth are 
calculated respectively ${ }^{[9,11]}$ :

$$
\begin{gathered}
\Delta f_{\gamma}=\frac{C_{m}^{*}-C_{m}^{e q}}{C_{m}^{e q}\left(k_{\gamma}-1\right)} \\
\Delta f_{\mathrm{Gr}}=G_{\mathrm{Gr}} \cdot \frac{\bar{C}_{m}^{*}-C_{m}^{e q}}{C_{\mathrm{Gr}}-C_{m}^{e q}}
\end{gathered}
$$

where $\Delta f_{\gamma}$ and $\Delta f_{\mathrm{Gr}}$ are the increment of solid fraction of austenite and graphite at each time step. $C_{m}^{e q}$ is the equilibrium concentration. $C_{m}^{*}$ is the actual liquid composition at the interface, the value of which is obtained by Eq. 1. The subscript $m$ represents the liquid/austenite interface cells in Eq. 3, which calculates the solid fraction of austenite at each time step. Similarly, the $m$ indicates the liquid/graphite or austenite/ graphite interface cells in Eq. 4, which is the governing equation for graphite growth. $G_{\mathrm{Gr}}$ and $\bar{C}_{m}^{*}$ are the parameters that are introduced to the equation to eliminate the anisotropy during the solidification process of graphite. The former is the geometrical parameter and the latter is the average concentration taken from the actual concentration of surrounding cells. The equilibrium concentration $C_{m}^{e q}$ is defined by ${ }^{[12]}$ :

$$
C_{m}^{e q}=C_{0}+\frac{T-T_{0}}{M_{i}}+\frac{\Gamma_{i} K_{i} f\left(\varphi, \theta_{0}\right)}{M_{i}}
$$

where $T_{0}$ is the equilibrium liquidus temperature at the initial composition $C_{0}$. $T$ is the temperature of the interface cells. $\Gamma_{i}$ is the Gibbs-Thomson coefficient and $M_{i}$ is the liquidus slope of austenite or graphite. $K_{i}$ is the mean curvature and a counting method is recommended to get this value ${ }^{[5]} . f\left(\varphi, \theta_{0}\right)$ is an anisotropic function of surface tension and its value is equal to 0 when the equilibrium concentration of graphite is calculated because of its isotropic characteristics.

The geometrical parameter and average concentration for a graphite interface cell is calculated with the following expression $^{[11]}$ :

$$
\begin{gathered}
G_{\mathrm{Gr}}=I_{M} \cdot \sum_{j=1}^{4} M_{j}+I_{N} \cdot \sum_{j=1}^{4} N_{j} \\
\left(\bar{C}_{m}^{*}\right)_{K}=\frac{\sum_{n=1}^{N}\left(\bar{C}_{m}^{*}\right)_{K-1}}{N}
\end{gathered}
$$

where $I_{M}$ and $I_{N}$ are the factors related to the types of neighboring cells. $M_{j}$ and $N_{j}$ represent the influence factors of the four nearest and four second-nearest neighboring cells. $K$ is the number of times to homogenize the concentration whose value is determined to be $8 . N$ is the number of interface cells which belong to the same "parent" graphite cell.

\section{Simulation results and discussion}

\subsection{Morphology evolution of graphite nodule}

To better determine the growth parameters, a single graphite nodule is simulated in the liquid phase. In the study of the effect of geometrical parameter on the morphology of graphite, assuming the influence factor of the nearest neighboring cells $I_{M}$ equals to 0.5 . Obviously, the role of $I_{N}$ is less important than $I_{M}$. Thus, the gradient values of $0,0.1,0.2,0.3,0.4$ are taken for $I_{N}$ in this study. Figure 1 indicates the simulation results under different values of $I_{N}$ at the same solidification time. It can be seen from the figure, when the value equals to 0.1 , the morphology of graphite nodule is most similar to the experimental result in Fig. 1(f). After obtaining the appropriate
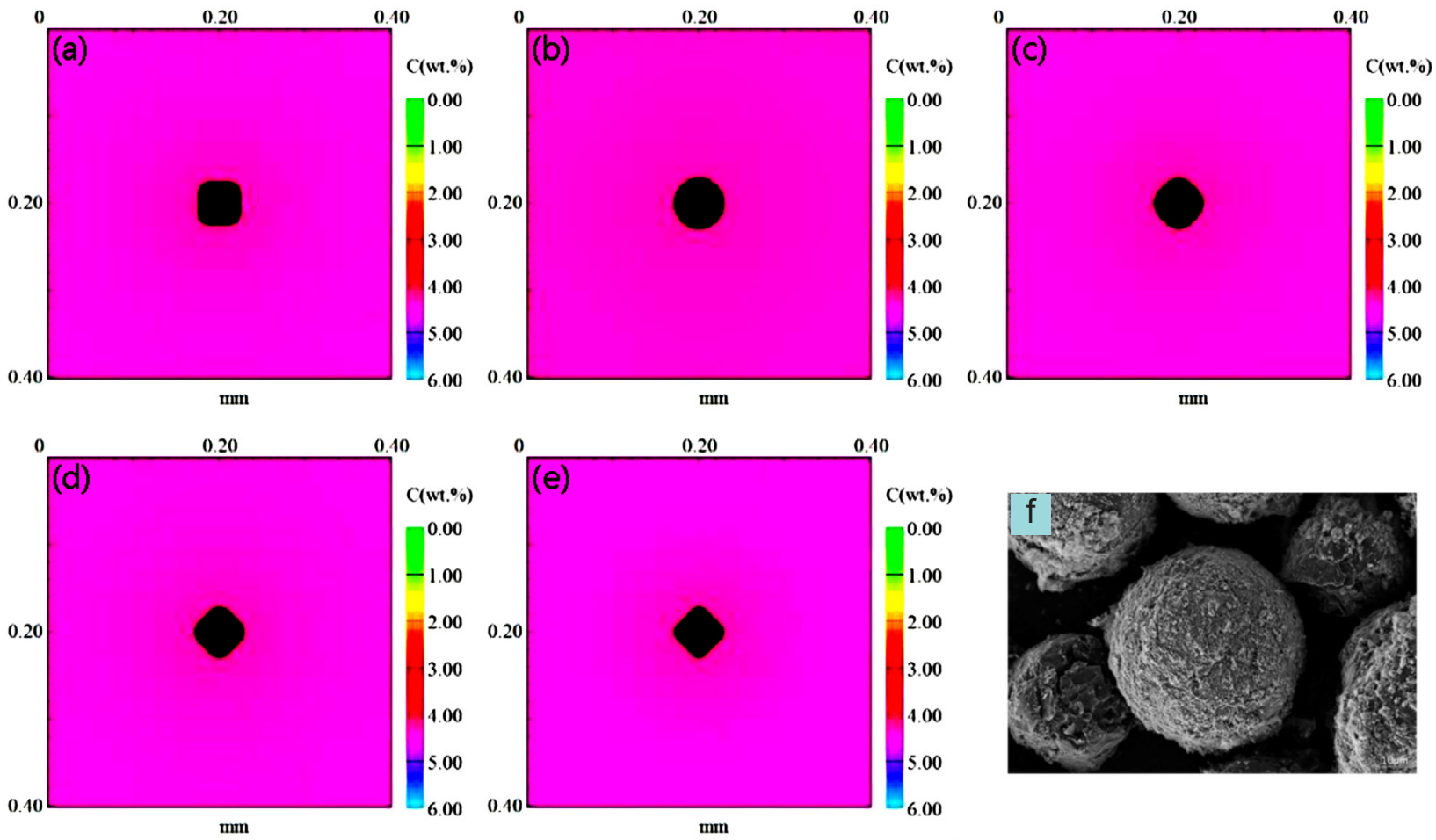

Fig. 1: Morphologies of graphite nodule under different values: (a) 0.0 , (b) 0.1 , (c) 0.2 , (d) 0.3 , (e) 0.4 , (f) SEM micrograph of graphite nodules 
parameters, the interactive growth of two graphite nodules was simulated using this model to investigate the impact of the growth environment. Figure 2 demonstrates the evolution of the growth of two graphite nodules. At the beginning of solidification, the graphite nodule A and B have a roughly spherical shape. With the extension of solidification time, the graphite A near the side of B grows slowly as a result of the depletion of solute carbon. Moreover, the growth of graphite A also inhibits the growth of $B$. Thus the shape of graphite nodules becomes irregular. The similar result can be observed through scanning electron microscope (SEM) in Fig. 2(d).

\subsection{Growth of hypoeutectic and hypereutectic SGI}

Studies on the solidification of hypoeutectic and hypereutectic SGI were carried out in order to better clarify and understand the proposed model. The simulation area was divided into $201 \times 201$ cells with a cell size of $2 \mu \mathrm{m}$. The initial concentration was set to 4.6wt.\%C for hypereutectic SGI and 4.06wt.\%C for hypoeutectic SGI. Figure 3 demonstrates the microstructure evolution of hypereutectic SGI. From Figs. 3(a) and (b), when the temperature drops down to liquidus, the nuclei of graphite are randomly formed in the melt and then grow in a spherical shape. Then austenite

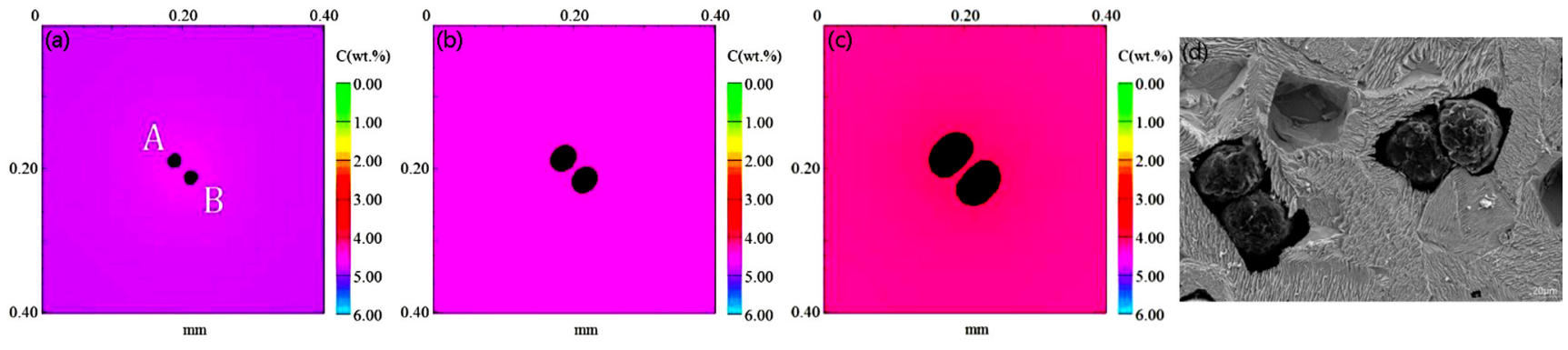

Fig. 2: Morphology evolution of two graphite nodules at different times: (a) $10 \mathrm{~s}$, (b) $20 \mathrm{~s}$, (c) $30 \mathrm{~s}$, (d) SEM micrograph of graphite nodules

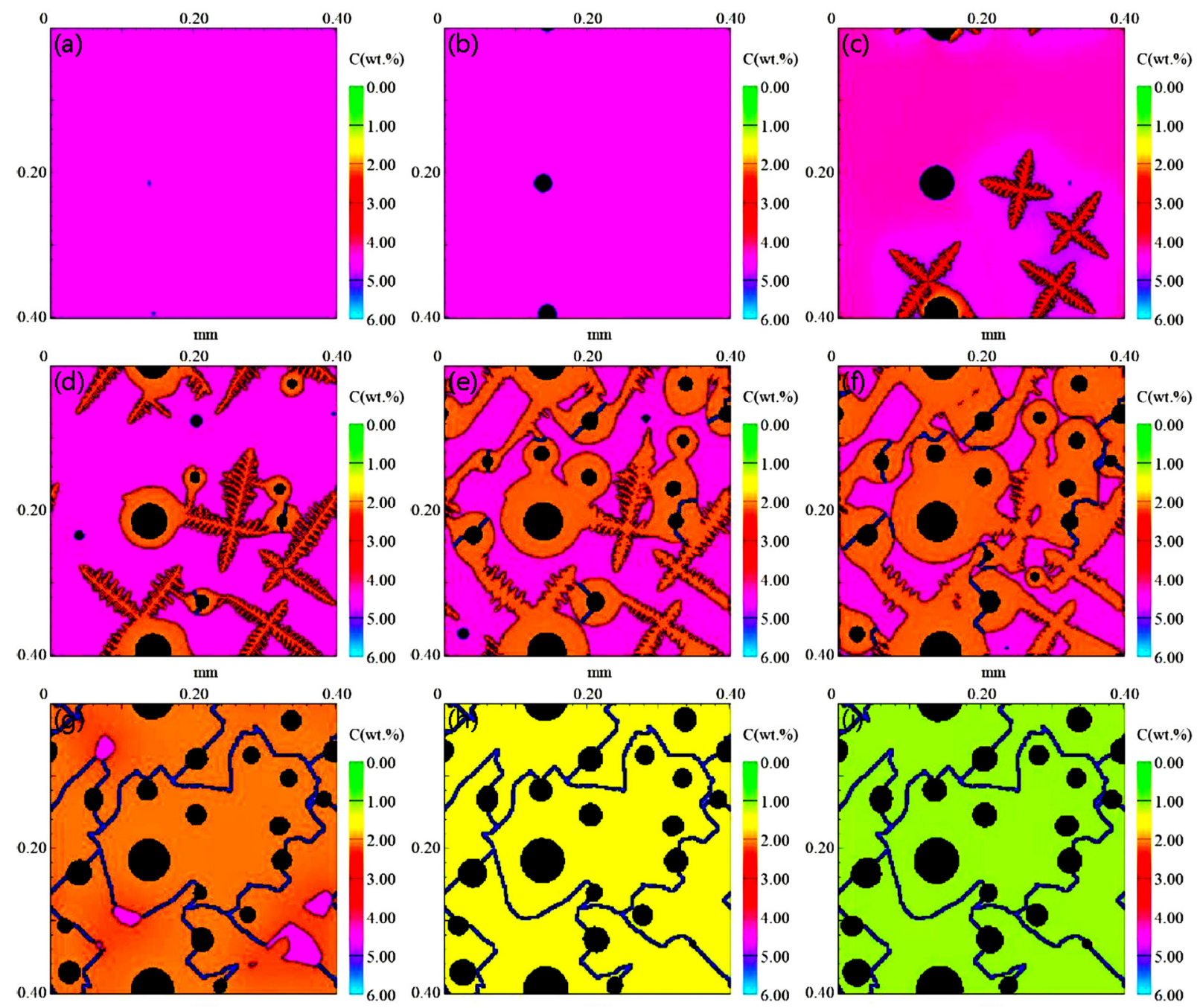

Fig. 3: Microstructure evolution of hypereutectic SGI 
dendrites begin to nucleate and grow when the temperature is lower than the eutectic temperature (Fig. 3c). Quickly, as shown in Fig. 3(d-f), austenite dendrites are moving closer to the carbondepleted zones which is caused by the growth of primary graphite and then envelop the graphite nodules. Meanwhile, eutectic graphite nodules precipitate and they are encapsulated in the austenite subsequently. The further growth of graphite requires the diffusion of carbon through the passage of surrounding austenite shells from the liquid. However, the diffusion coefficient of carbon in the austenite is small ${ }^{[9]}$, the growth of encapsulated graphite nodules is slower than in the liquid phase. At the end of the solidification, austenite dendrites grow in the liquid phase and get in touch with each other as shown in Fig. 3(g).

Figure 4 depicts the changes of temperature, total solid fraction, austenite and graphite solid fraction over time corresponding to the simulation process of hypereutectic SGI in Fig. 3. At the beginning, in Fig. 3(a-b), a few number of graphite lead to the fact that the latent heat of solidification has little effect on the temperature of the system. Then the precipitation of eutectic austenite and eutectic graphite in Fig. 3(c) will release a lot of latent heat, making the temperature recover. At the stage of Fig. 3(d-g), the eutectic platform appears due to the balance between the rise of temperature caused by latent heat and the drop of temperature caused by heat transfer. And the temperature continues to decline when the eutectic solidification is over as can be seen from Fig. 4. In addition, it can be concluded from Fig. 3(h-i) that there is no liquid phase to provide solute carbon for the growth of graphite. It is necessary to absorb carbon from the austenite, resulting a significant decrease in the solid fraction of austenite and an increase in the solid fraction of the graphite as shown in Fig. 4.

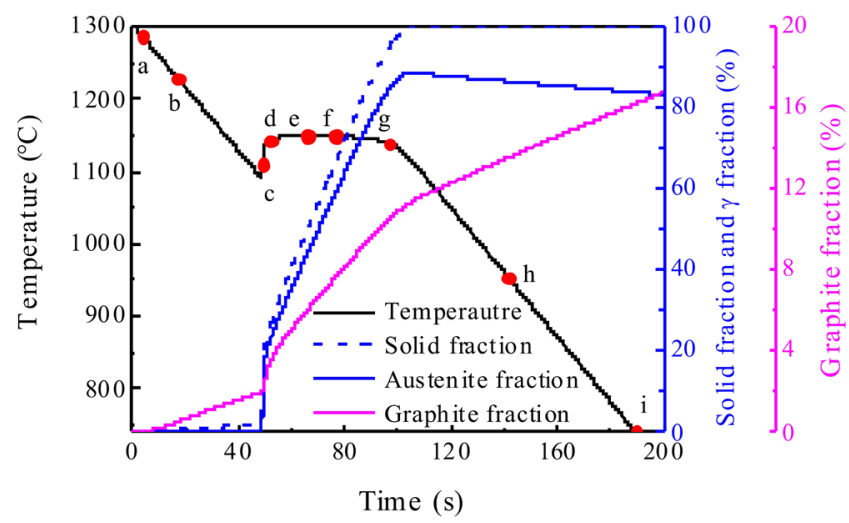

Fig. 4: Changes of temperature, total solid fraction, austenite and graphite solid fraction over time for hypereutectic SGI

Figure 5 shows the microstructure evolution of hypoeutectic SGI. Different from the solidification of hypereutectic SGI, in Fig.5(a-b), when the temperature reaches the liquidus temperature, the solidification starts with the nucleation of austenite and growth in dendrites along the preferential growth direction. Once the eutectic solidification conditions are satisfied, eutectic graphites precipitate from the liquid in Fig. 5(c). Similar to the eutectic solidification process of hypereutectic SGI, the growing graphite nodules is soon surrounded by austenite in Fig. 5(d-g). And the further growth of eutectic graphite also requires the diffusion of carbon through the passage of surrounding austenite shells from the liquid. Similarly, austenite dendrites contact each other at the end of the solidification as shown in Fig. 5(h-i). Figure 6 indicates the changes of temperature, total solid fraction, austenite and graphite solid fraction over time corresponding to the simulation process of hypoeutectic SGI in Fig. 5. And they have the similar regularity with the hypereutectic SGI in Fig. 4.

\section{Conclusions}

(1) The concentration averaging and geometrical parameter are recommended to establish a new graphite growth model. By selecting appropriate parameters, the improved model can simulate the growth of a single graphite nodule and the morphology evolution of two adjacent graphite nodules. It was found that the graphite grows slowly when its surrounding carbon concentration is low.

(2) To simulate the solidification process of typical hypoeutectic and hypereutectic SGI, the growth model of graphite and austenite is coupled, and the calculation methods of temperature field, concentration field, the increase of austenite and graphite solid fraction are described in detail. The simulated results indicate that for the graphite, when it is the primary phase, the growth is controlled by the diffusion of carbon in the liquid phase. When the eutectic conditions are met, the graphite nodules are encapsulated in the austenite dendrites quickly. At the beginning, the solute carbon required for the growth of graphite is mainly transported through the austenite shell. At the end of the eutectic solidification, the graphite nodules will continue to grow by absorbing carbon from the austenite. By analyzing the changes of concentration, temperature and microstructure over time, this model can reasonably reproduce the microstructure evolution of SGI during solidification.

\section{References}

[1] Guo J, Samonds M T. Modeling and Experimental Validation of Ductile Iron Castings During Solidification. Journal of Materials Engineering and Performance, 2008, 17(6): 831-837.

[2] Stefanescu D M. Solidification and modeling of cast iron - A short history of the defining moments. Materials Science and Engineering A, 2005, 413: 322-333.

[3] Zheng Hongliang. Solidification simulation of spheroidal graphite iron based on macro-micro models. Dissertation, Jinan: Shandong University, 2007. (In Chinese)

[4] Rappaz M, Gandin C A. Probabilistic modelling of microstructure formation in solidification processes. Acta Metallurgica et Materialia, 1993, 41(2): 345-360.

[5] Nastac L. Numerical modeling of solidification morphologies and segregation patterns in cast dendritic alloys. Acta 

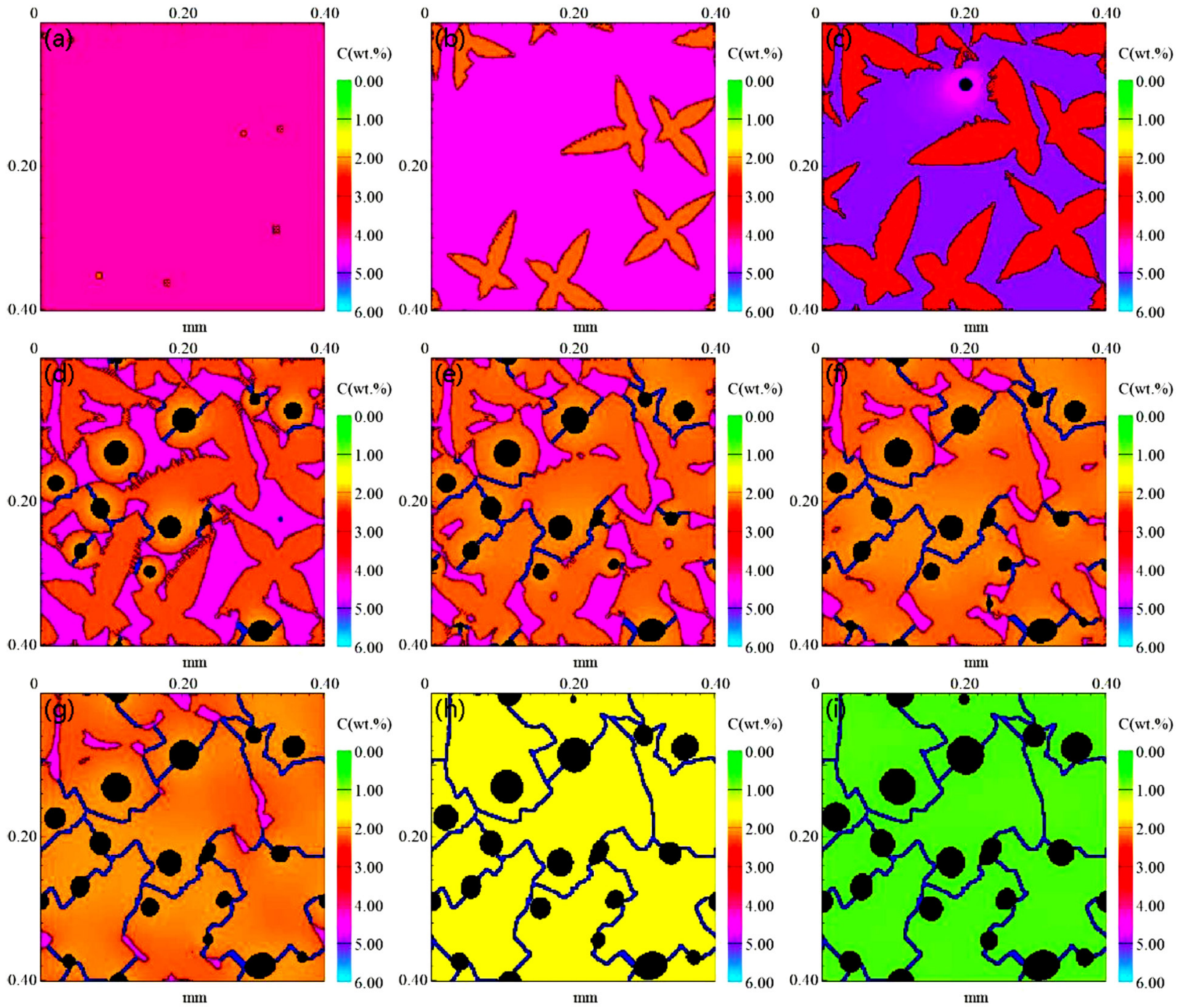

Fig. 5: Microstructure evolution of hypoeutectic SGI

Materialia, 1999, 47(17): 4253-4262.

[6] Beltran-Sanchez L, Stefanescu D M. Growth of solutal dendrites: a cellular automaton model and its quantitative capabilities. Metallurgical and Materials Transactions A, 2003, 34(2): 367-382.

[7] Beltran-Sanchez L, Stefanescu D M. A quantitative dendrite growth model and analysis of stability concepts. Metallurgical and Materials Transactions A, 2004, 35(8): 2471-2485.

[8] Wang W, Lee P D, Mclean M. A model of solidification microstructures in nickel-based superalloys: predicting primary dendrite spacing selection. Acta Materialia, 2003, 51(10): 2971-2987.

[9] Zhu M, Zhang L, Zhao H, et al. Modeling of microstructural evolution during divorced eutectic solidification of spheroidal graphite irons. Acta Materialia, 2015, 84: 413-425.

[10] Chen R, Xu Q, Liu B. Modeling of aluminum-silicon irregular eutectic growth by cellular automaton model. China Foundry, 2016, 13(2): 114-122.

[11] Zhao Qingming. Numerical Simulation on Spheroidal Graphite Cast Iron Solidification Process with Cellular Automaton. Dissertation, Jinan: Shandong University, 2016. (In Chinese)

[12] Chen R, Xu Q, Liu B. A modified cellular automaton model for

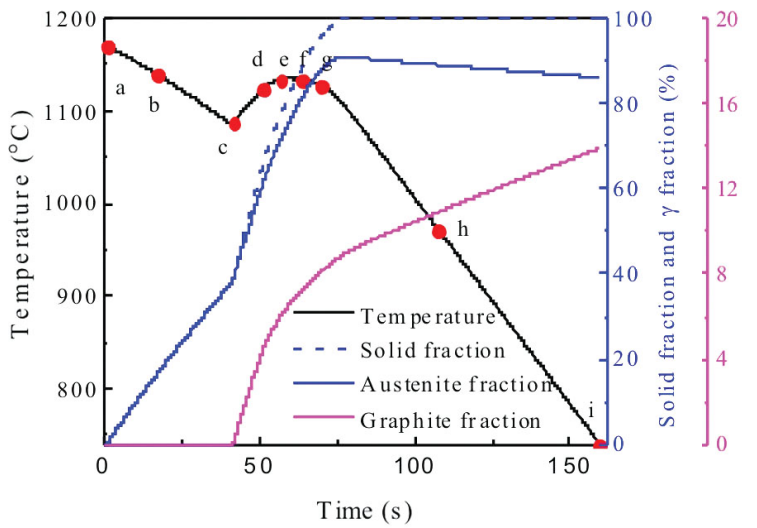

Fig. 6: Changes of temperature, total solid fraction, austenite and graphite solid fraction over time for hypoeutectic SGI

the quantitative prediction of equiaxed and columnar dendritic growth. Journal of Materials Science \& Technology, 2014, 30(12): 1311-1320. 